\title{
Controlling the morphology of copper-silica nanocomposites from laser ablation in liquid
}

\author{
Mallory G. John and Katharine Moore Tibbetts \\ Department of Chemistry, Virginia Commonwealth University, Richmond, VA 23284, USA
}

\begin{abstract}
Synthesis of copper-silica nanocomposites with controllable morphology and composition were produced with a one-step femtosecond reactive laser ablation in liquid (fs-RLAL) technique. The composite nanomaterials were generated by focusing femtosecond near-IR laser pulses onto a silicon wafer immersed in an aqueous copper(II) nitrate solution, with the solution $\mathrm{pH}$ adjusted using nitric acid or potassium hydroxide. Under acidic conditions (pH 3.0 and 5.4), little copper was incorporated in the predominantly silica product (1.4 and 1.5 wt.\%). These acidic conditions yielded large $\sim 30-80 \mathrm{~nm}$ silica particles, with some particles consisting of copper core/silica shell. In contrast, increasing the solution $\mathrm{pH}$ to 10.4 resulted in extremely high $\mathrm{Cu}$ loading of $31.5 \mathrm{wt} . \%$ and a composite product consisting of $1.5 \mathrm{~nm}$ copper clusters distributed throughout a matrix of amorphous silica and copper phyllosilicate. The relationship between the precursor solution $\mathrm{pH}$ and the product morphology and copper loading is attributed to the point of zero charge (PZC) of silica, in which the high solution $\mathrm{pH}$ allows for electrostatic adsorption to occur between the deprotonated silica clusters from the ablated silicon wafer and the copper hydroxide dimer formed in solution.

Keywords: reactive laser ablation in liquid, femtosecond laser, copper-silica nanocomposite, copper phyllosilicate
\end{abstract}

\footnotetext{
${ }^{1}$ kmtibbetts@vcu.edu
} 


\section{Introduction}

Copper nanoparticles (Cu NPs) are valued for their low cost, high conductivity, and thermal stability, making them a popular alternative to rare earth metals for biological sensing and imaging [1], antimicrobial applications [2], inkjet-printable electronics [3], and catalysis [4]. In particular, the ability of copper to access many oxidation states makes supported Cu NPs active catalysts towards reactions such as electrochemical reduction [5], thermochemical hydrogenation [6] and photochemical reduction [7] of $\mathrm{CO}_{2}$, photocatalytic degradation of organic dyes [8], and other organic transformations [9-11].

While $\mathrm{Cu}$ NPs possess high catalytic activity and high temperature stability, a major bottleneck to using copper-based nanomaterials for catalysis is the propensity for small $\mathrm{Cu}$ NPs to agglomerate, and for $\mathrm{Cu}$ surfaces to oxidize. Support materials such as graphene, oxides, polymers, and metal-organic frameworks (MOFs) are added to prevent agglomeration and surface oxidation, while preserving the high catalytic activity of $\mathrm{Cu}$ NPs $[4,12,13]$. In particular, silica has been used as a support material for various metal NPs due to the silanol surface groups that enhance binding with metal NPs $[4,14]$. The majority of synthetic approaches to fabricating copper-silica nanostructures involve wet chemical methods such as incipient wetness impregnation, deposition-precipitation, strong electrostatic adsorption, and ammonia evaporation [4, 5, 11, 13-15]. In these methods, the silica is either prepared by the Stöber method or purchased commercial amorphous/fumed silica, and the copper is added in the form of a salt complex. Copper-silica bonding is achieved by heating up the slurry or solution, followed by calcining the finished product. A drawback of many of these methods is that uneven distribution of the copper complex throughout the silica often results in poorly dispersed $\mathrm{Cu}$ NPs with large size distributions and low copper loading.

Laser ablation in liquid (LAL) has recently emerged as a robust alternative synthesis route to myriad (supported) NPs that can overcome many challenges inherent in wet-chemical synthesis [16-19]. LAL involves focusing intense laser 
pulses onto a solid target immersed in liquid, which produces a localized plasma at the solid-liquid interface containing reactive electrons, radicals, and ions [20]. The plasma reaches transient temperatures exceeding $5000 \mathrm{~K}$ that cool on submicrosecond timescales due to the surrounding ambient liquid [21]. These highly nonequilibrium conditions generate NPs comprised of the target material with exotic metastable phases and bonding environments that are stable without added capping agents, making them ideal for catalysis applications [16-18]. Because LAL generates nanomaterials under ambient conditions in water, it is considered a 'green' and sustainable synthesis method [16, 22]. LAL of a $\mathrm{Cu}$ target in various solvents have been widely used to synthesize $\mathrm{Cu}$ NPs with solvent- and laser-dependent oxidation states and morphologies [23-26].

When LAL is carried out in a solution containing metal ions or other species that interact with the ablated target atoms, the technique is referred to as Reactive Laser Ablation in Liquid (RLAL) [16]. The first demonstration of RLAL in 2008 produced Ag and Au NPs by ablating a silicon wafer immersed in either $\mathrm{Ag}\left(\mathrm{NO}_{3}\right)$ or $\mathrm{HAuCl}_{4}$ aqueous solutions [27]. Since this initial work, many metastable and unique bi- and multi-metallic nanostructures have been reported. For instance, mixed-metal Pt-Co and Pt-Co-Cu oxide NPs for fuel cell applications were synthesized by ablating a Co target immersed in Pt and $\mathrm{Cu}$ metal salt solutions [28-30], and Ni-Fe layered hydroxides doped with $\mathrm{Ti}$ and La for electrochemical water splitting were synthesized by ablation of $\mathrm{Fe}$ powder in aqueous solutions of $\mathrm{Ni}, \mathrm{Ti}$, and La salts [31,32]. Complex metaloxide mineral phases of copper and zinc were synthesized by RLAL of $\mathrm{Zn}$ or $\mathrm{Cu}$ targets immersed in aqueous $\mathrm{Zn}$ or $\mathrm{Cu}$ salt solutions [33], and silica-supported $\mathrm{Au}$ or $\mathrm{Ag}$ nanomaterials have been synthesized by ablating silicon wafers in aqueous gold or silver salt solutions [27, 34-36]. Recently, we generated sub-3 $\mathrm{nm} \mathrm{Au}$ NPs dispersed throughout a silica matrix by ablating a silicon wafer immersed in a $\left[\mathrm{AuCl}_{4}\right]^{-}$solution using femtosecond laser pulses [36].

In this work we report the synthesis and characterization of copper-silica nanocomposites generated from a femtosecond-RLAL (fs-RLAL) technique, in which fs laser pulses are focused onto a silicon wafer immersed in $\mathrm{Cu}\left(\mathrm{NO}_{3}\right)_{2}$ 
solutions under three $\mathrm{pH}$ conditions. The product morphology and $\mathrm{Cu}$ loading on the silica were strongly dependent on the precursor solution $\mathrm{pH}$, with the highest copper loading achieved at $\mathrm{pH}$ 10.4. This $\mathrm{pH}$-dependent copper loading was reflected in the catalytic activity of the samples, determined using the model reaction of catalytic para-nitrophenol reduction by sodium borohydride [37]. We will discuss the role that the solution $\mathrm{pH}$ plays on the surface charge of ablated silica species, and how surface interactions drive the formation of different morphology and wt.\% loading of $\mathrm{Cu}$ in the products.

\section{Materials and Methods}

\subsection{Materials}

Silicon wafers (n-doped, (111)-oriented, single side polished, $300 \mu \mathrm{m}$ thick, NOVA electronic materials), copper(II) nitrate, $\mathrm{Cu}\left(\mathrm{NO}_{3}\right)_{2}$ (Fisher), potassium hydroxide, $\mathrm{KOH}$ (Fisher), nitric acid, $\mathrm{HNO}_{3}$ (Fisher), sodium borohydride, $\mathrm{NaBH}_{4}$ (Acros Organics), and para-nitrophenol, PNP (Acros Organics) were used as received. Stock and working solutions were prepared with purified wa-

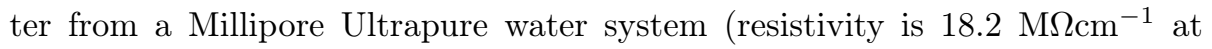
$\left.25^{\circ} \mathrm{C}\right)$.

\subsection{Sample Preparation}

Working solutions of $\mathrm{Cu}\left(\mathrm{NO}_{3}\right)_{2}(2.0 \mathrm{mM})$ were prepared from a freshly prepared aqueous stock solution $(50 \mathrm{mM})$ and the $\mathrm{pH}$ was recorded as $\sim 5.4$. Either $\mathrm{HNO}_{3}(1.0 \mathrm{mM})$ was added from a $10 \mathrm{mM}$ stock solution or $\mathrm{KOH}(5.0 \mathrm{mM})$ was added from a $200 \mathrm{mM}$ stock, resulting in working solution $\mathrm{pH}$ values of 3.0 and 10.4, respectively. The working solution was transferred to a quartz cuvette $(3 \mathrm{~mL})$ equipped with a stir bar, and a pre-cut silicon wafer was placed in the cuvette and secured to one side.

fs-RLAL irradiation of the Si wafer immersed in the working solution was conducted for 30 min while stirring (details of laser parameters in section 2.4), followed by centrifugation for $15 \mathrm{~min}$ at 6,000 rpm (Thermo Fisher AccuSpin 
Micro 17). The supernatant was replaced with water and centrifuged a second time for $15 \mathrm{~min}$ at 6,000 rpm. The resulting pellet was collected for either characterization or redispersed in water and tested for its catalytic activity. The following naming convention was used for all samples: $\mathrm{Cu}$-silica-[solution $\mathrm{pH}$. For example, Cu-silica-3.0 corresponds to the sample containing $2.0 \mathrm{mM}$ $\mathrm{Cu}\left(\mathrm{NO}_{3}\right)_{2}$ and $1.0 \mathrm{mM} \mathrm{HNO}$, with a $\mathrm{pH}$ of 3.0. Table 1 displays the sample names and solution compositions for clarity.

\begin{tabular}{c|cc}
\hline Sample & Solution Composition $^{a}$ & Initial Solution $\mathrm{pH}$ \\
\hline Cu-silica-3.0 & $2 \mathrm{mM} \mathrm{Cu}\left(\mathrm{NO}_{3}\right)_{2}+1 \mathrm{mM} \mathrm{HNO}_{3}$ & $3.0 \pm 0.1$ \\
Cu-silica-5.4 & $2 \mathrm{mM} \mathrm{Cu}\left(\mathrm{NO}_{3}\right)_{2}$ & $5.4 \pm 0.1$ \\
$\mathrm{Cu}$-silica-10.4 & $2 \mathrm{mM} \mathrm{Cu}\left(\mathrm{NO}_{3}\right)_{2}+5 \mathrm{mM} \mathrm{KOH}$ & $10.4 \pm 0.2$ \\
\hline
\end{tabular}

Table 1: Sample, solution composition, and initial solution $\mathrm{pH}$ values for experiments. ${ }^{a}$ all solution prepared in DI water.

\subsection{Catalytic Reduction of Para-nitrophenol}

The catalytic reduction of para-nitrophenol (PNP) by $\mathrm{NaBH}_{4}$ was carried out in a home-built in situ UV-vis spectrometer (details in section 2.4). In a typical catalytic run, the collected pellet (details in section 2.2) was dispersed in water $(3.0 \mathrm{~mL})$ and $300 \mu \mathrm{L}$ of this solution was added to a cuvette containing PNP $(0.1 \mathrm{mM})$ and $\mathrm{NaBH}_{4}(10 \mathrm{mM})$ while stirring. The PNP was added from a stock solution $(1.5 \mathrm{mM})$ and the $\mathrm{NaBH}_{4}$ was added from a freshly prepared stock solution $(100 \mathrm{mM})$. The absorbance at $400 \mathrm{~nm}$ (as the para-nitrophenolate ion) was recorded, and the reaction was considered complete when it had disappeared completely. Data processing for the PNP reaction was conducted based on the methods of Ref. [38] and details are provided in the Supplemental Information (Fig. S1).

\subsection{Instrumentation}

The experimental laser setup has been described in detail in Refs. [36, 39, 40]. Briefly, a Ti:Sapphire regenerative amplifier delivering $7 \mathrm{~mJ}, 30 \mathrm{fs}$ 
pulses with a bandwidth centered at $800 \mathrm{~nm}$ and a repetition rate of $1 \mathrm{kHz}$ was attenuated to $200 \mu \mathrm{J}$ for the ablation experiments. The $10 \times 10 \times 40 \mathrm{~mm}$ quartz fluorescence cuvette containing the pre-cut silicon wafer was placed 10 $\mathrm{mm}$ before the focal point of a $f=50 \mathrm{~mm}$ lens. The converging beam propagates through $9.7 \mathrm{~mm}$ of liquid to reach the $0.3 \mathrm{~mm}$ thick Si wafer. We note that no white light was generated on the front face of the cuvette because the large initial beam diameter of $11 \mathrm{~mm}$ and high numerical aperture of the focusing lens were sufficient to eliminate any nonlinear optical effects until a few $\mathrm{mm}$ before the Si-liquid interface. The spot size on the wafer was $85 \mu \mathrm{m}$ diameter, measured using an optical microscope of an ablated Si wafer. Under these conditions, the laser fluence was $3.5 \mathrm{~J} \mathrm{~cm}^{-2}$ and peak intensity was $1.17 \times 10^{14} \mathrm{~W} \mathrm{~cm}^{-2}$. The cuvette was placed on a micro-stir plate (Thermo Scientific) mounted on x- and y- motorized translation stages (Thorlabs), which moved in a zig-zag pattern at a velocity of $0.5 \mathrm{~mm} / \mathrm{s}$.

PNP catalysis runs were carried out in a home-built in situ UV-vis spectrometer, with a deuterium-tungsten light source (Ocean Optics, DH2000-DUV), optical fibers, a sample holder for $10 \times 10 \times 40 \mathrm{~mm}$ cuvettes placed on a stir plate (300 rpm stir rate), and a compact spectrometer (Ocean Optics HR4000). Spectra were collected every 1.2 seconds using LabVIEW software (National Instruments).

\subsection{Characterization}

Transmission Electron Microscopy (TEM) TEM images were collected on a JEOL JEM-1230 TEM at $120 \mathrm{kV}$. High resolution TEM (HRTEM) images and SAED patterns were collected on an FEI Titan $80-300 \mathrm{kV}$ with a Gatan 794 Multi-Scan Camera. Samples were prepared by drop-casting the diluted pellet onto a carbon-coated copper grid (100 mesh, Ted Pella, Inc.) and left to dry for at least $24 \mathrm{hr}$ at room temperature. Size distributions were determined by measuring 300 individual particles from three separate parts of the grid using ImageJ software. Gatan Microscopy Software Suite version 3.x was used to determine the crystal lattices of the nanoparticles in the HRTEM images. 
Details on this process are included in the Supplemental Information (Fig. S2).

Scanning Electron Microscopy-Energy Dispersive X-ray Spectroscopy (SEM-EDX) SEM-EDX was carried out on a Hitachi FE SEM SU-70 (spatial resolution $1.0 \mathrm{~nm}$ ) equipped with an Energy Dispersive X-ray Spectroscopy (EDX) detector. Images were obtained at $10 \mathrm{keV}$ and elemental analysis was conducted at $15 \mathrm{keV}$, with ZAF standardless quantification employed for EDS measurements. Samples were prepared by drop casting the centrifuged pellets onto conductive carbon tape stabilized on an aluminum stage, and drying under vacuum at room temperature.

X-ray Photoelectron Spectroscopy (XPS) XPS was conducted on a PHI VersaProbe III Scanning XPS Microprobe with a monochromatic Al k $\alpha$ X-ray source $(1486.6 \mathrm{eV})$ run at $25 \mathrm{~W}$ and $15 \mathrm{KV}$, with a pass energy set to $112 \mathrm{eV}$ for survey scans and $69 \mathrm{eV}$ for high resolution spectra. A spot diameter of $200 \mu \mathrm{m}$ was irradiated using a take off angle of $90^{\circ}$, and a detector was situated at an angle of $45^{\circ}$. Charge neutralization was achieved by employing an ion gun and a flood gun during the analysis. Samples were prepared by drop casting the centrifuged pellet onto conductive carbon tape. Sample analysis was carried out using CasaXPS Software version 2.3.19PR1.0, employing Gaussian and Lorentzian convolution to fit the spectral lines, and all high resolution spectra were corrected by shifting the C1s peak at $284.8 \mathrm{eV}$.

X-ray Diffraction (XRD) XRD was conducted on a Panalytical Empryrean Diffractometer with $\mathrm{CuK} \alpha$ radiation $(\lambda=0.15418 \mathrm{~nm})$ at $40 \mathrm{kV}$ and $45 \mathrm{~mA}$, with scanning angle $(2 \theta)$ of $10-90^{\circ}$ and a gonio focusing geometry. Samples were prepared for XRD analysis by drying the centrifuged pellet under vacuum at room temperature.

Fourier Transform Infrared Spectroscopy (FTIR) FTIR analysis was conducted on a Nicolet iS50 FTIR spectrometer equipped with a mid- and farIR-capable diamond ATR. Spectra were obtained using 32 scans in the range of 4000 to $400 \mathrm{~cm}^{-1}$ with $5 \mathrm{~cm}^{-1}$ resolution. Samples were prepared for FTIR analysis by drying the centrifuged pellet under vacuum at room temperature. 


\section{Results}

\subsection{Characterization}

The three $\mathrm{Cu}$-silica products are visualized in the TEM images displayed in Fig. 1a-c with detailed insets, and additional TEM images are reported in the Supplemental Information (Fig. S3). While all three products contain

large spherical particles around $\sim 70-100 \mathrm{~nm}$ in diameter, these particles are most abundant in the $\mathrm{Cu}$-silica-3.0 sample, and rarely seen in the Cu-silica-10.4 sample. Many of the spherical particles in the $\mathrm{Cu}$-silica-3.0 and $\mathrm{Cu}$-silica-5.4 samples are smooth throughout the entire particle, while some have a darker core and lighter shell, indicative of a $\mathrm{Cu}$-core and silica-shell structure (insets in Fig. 1a and b). The Cu-silica-3.0 sample had very few core-shell particles. Size distribution analysis was possible on only 43 particles, and histograms of the core and outer diameter are displayed in Fig. S3 in the Supplemental Material. The Cu-silica-5.4 had substantially more core-shell particles than the $\mathrm{Cu}$-silica3.0 sample, with size distribution analysis displayed in Figure 1d.The inner core had a mean diameter of $22.4 \pm 14.4 \mathrm{~nm}$ with sizes ranging from $2-63 \mathrm{~nm}$, and the outer shell mean was $32.1 \pm 14.8 \mathrm{~nm}$ with sizes ranging $12-84 \mathrm{~nm}$ in diameter. The Cu-silica-10.4 sample exhibits completely different morphology from the samples produced at lower $\mathrm{pH}$ (Fig. 1c). This product predominantly contains small, $1.52 \pm 0.75 \mathrm{~nm} \mathrm{Cu}$ NPs dispersed throughout a matrix made up of long nano-needles and amorphous structures, along with a few large spherical particles decorated with small $\mathrm{Cu}$ NPs (inset). A histogram of the $\mathrm{Cu}$ NPs is displayed in Fig. 1e fit to a Gaussian distribution.

HRTEM images of the $\mathrm{Cu}$-silica-5.4 and $\mathrm{Cu}$-silica-10.4 products are displayed in Fig. 2a and c with SAED patterns (Fig. 2b and d). HRTEM analysis was not performed on the Cu-silica-3.0 sample due to the low number of coreshell particles. The inset in the Cu-silica-10.4 HRTEM image shows a small crystalline nanoparticle with lattice spacings measuring $2.13 \AA$ corresponding to the (200) plane of $\mathrm{Cu}_{2} \mathrm{O}$ [41]. The bottom inset of Fig. 2a shows the amorphous structure of the silica. The SAED pattern in Fig. $2 \mathrm{~b}$ has two faint diffraction 


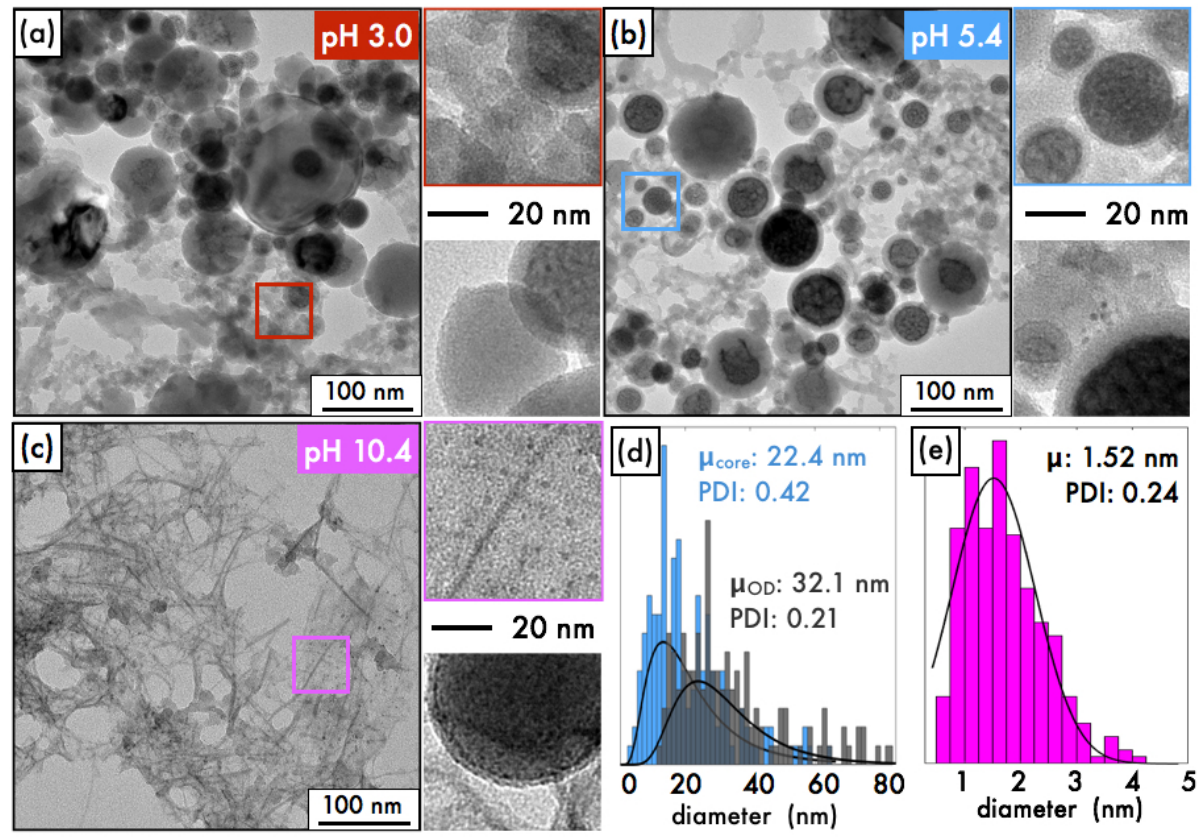

Figure 1: TEM images of Cu-silica samples at pH 3.0 (a), pH 5.4 (b), and pH 10.4 with histograms of Cu-silica-5.4 (e) and Cu-silica-10.4 (e). 
rings measuring $2.41 \AA$ and $2.04 \AA$ corresponding to the (111) plane of $\mathrm{Cu}_{2} \mathrm{O}$ and the (111) plane of fcc-Cu [42]. The Cu-silica-5.4 sample in Fig. 2c had some small crystalline nanoparticles with lattice spacings measuring $2.07 \AA$, corresponding to the (111) plane of fcc-Cu. It was difficult to confirm that the core of the core-shell particles was crystalline copper due to the thick amorphous layer over top of it (bottom inset in Fig. 2c). The SAED pattern of the $\mathrm{Cu}$ silica-5.4 sample in Fig. 2d shows three faint rings with diameters $3.11 \AA$, 2.01 $\AA$, and $1.14 \AA$, corresponding to the (111) plane of fcc-Si, the (111) and (311) planes of fcc- $\mathrm{Cu}$ [41]. Our results are consistent with the $\mathrm{Cu}$ crystalline phases generated by laser ablation of copper, in which the major phases of copper generated included $\mathrm{Cu}_{2} \mathrm{O}$ and fcc-Cu [23-25].
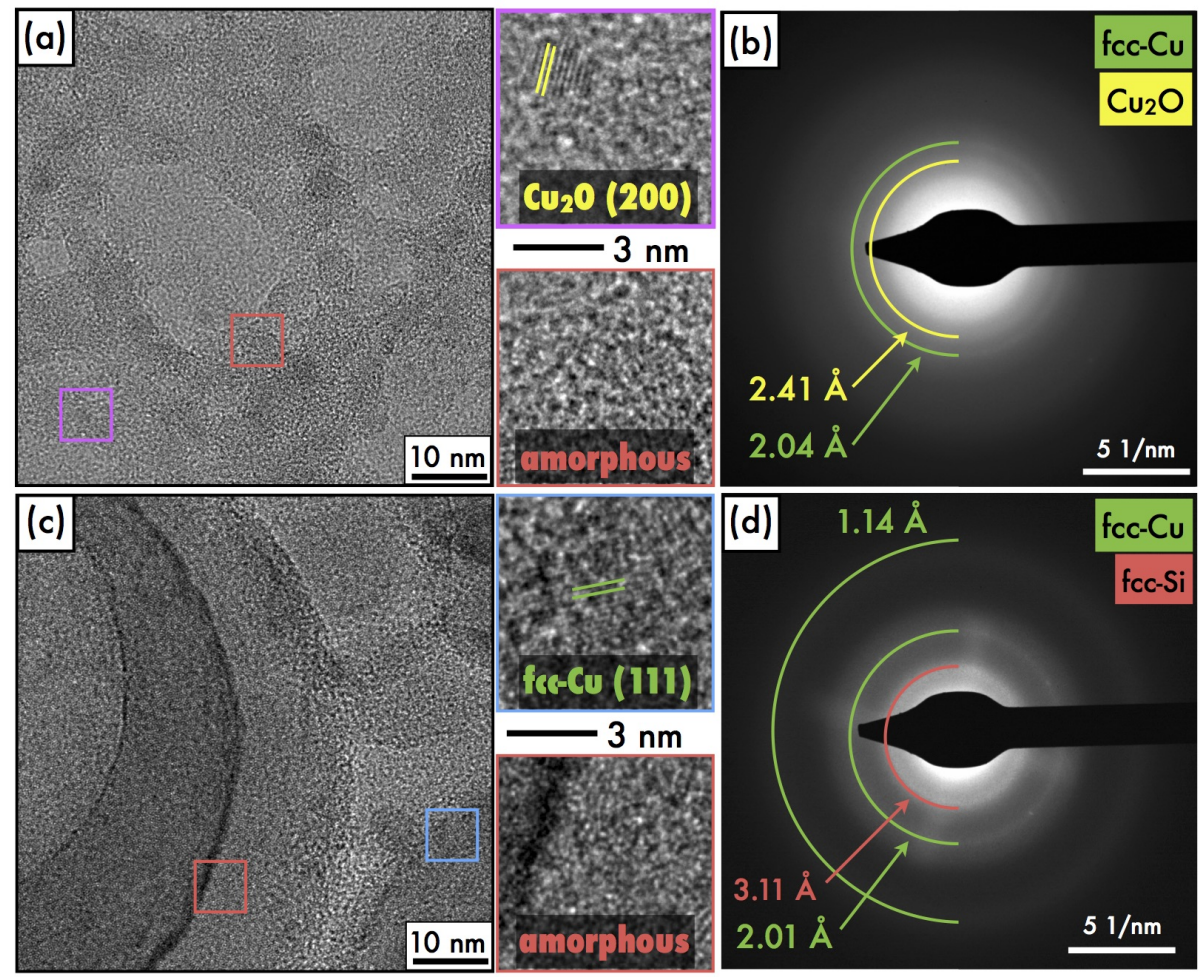

Figure 2: HRTEM images of Cu-silica-10.4 (a) and Cu-silica-5.4 (c) with SAED patterns (b) and (d), respectively. 
SEM-EDX analysis was performed on the three $\mathrm{Cu}$-silica samples, with a representative EDX spectrum of the $\mathrm{Cu}$-silica-10.4 sample displayed in Fig. 3. The peaks located at $0.525 \mathrm{keV}, 0.950 \mathrm{keV}$, and $1.74 \mathrm{keV}$ correspond to the $\mathrm{O}$ $\mathrm{K} \alpha, \mathrm{Cu} \mathrm{L} \alpha$, and $\mathrm{Si} \mathrm{K} \alpha$ lines. The inset graph shows the wt.\% $\mathrm{Cu}, \mathrm{Si}$, and $\mathrm{O}$ quantified in the samples, and Table 2 displays the numerical values of wt.\% $\mathrm{Cu}$, $\mathrm{Si}$, and $\mathrm{O}$ from EDX, XPS, and ICP-OES analysis. The Cu-silica-10.4 sample contains the highest amount of $\mathrm{Cu}$, about ten to twenty times the amount as the $\mathrm{Cu}$-silica-5.4 and $\mathrm{Cu}$-silica-3.0 samples. The significantly higher $\mathrm{Cu}$ loading in the $\mathrm{Cu}$-silica-10.4 sample is corroborated by the ICP-OES and XPS results, also displayed in Table 2. XPS data was converted from atomic \%, with calculations provided in Table S1 in the Supplemental Information. XPS analysis shows significant surface oxidation of the three samples, which is compensated for by the decrease in $\mathrm{Cu}$ content in the $\mathrm{Cu}$-silica-10.4 sample, and decrease in $\mathrm{Si}$ content in the other two samples. The copper content did not decrease between EDX and XPS analysis for the Cu-silica-5.4 sample, suggesting that the copper present within the top $10 \mathrm{~nm}$ of this sample is protected from surface oxidation.

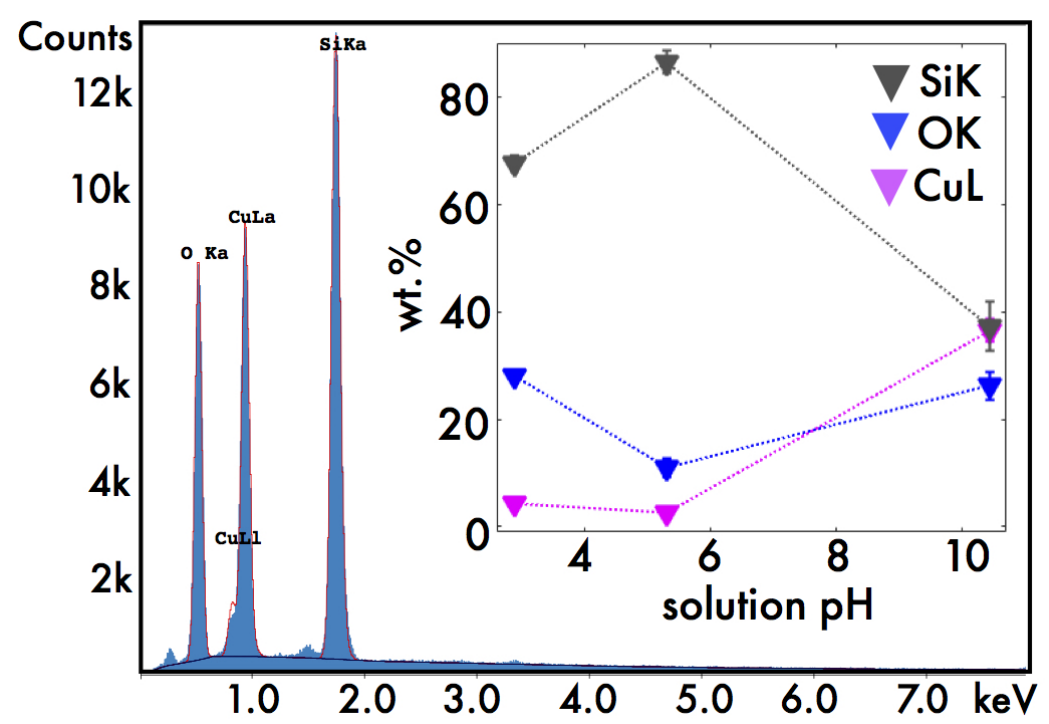

Figure 3: SEM-EDX spectrum of representative Cu-silica-10.4 sample with inset of wt.\% of $\mathrm{SiK}, \mathrm{OK}$, and CuL for different $\mathrm{pH}$ solutions. 


\begin{tabular}{c||c|ccc|ccc}
\hline \multicolumn{1}{c}{ ICP-OES $^{a}$} & \multicolumn{3}{c}{ SEM-EDX $^{a}$} & \multicolumn{3}{c}{ XPS $^{b c}$} \\
\hline Sample & $\mathrm{Cu}$ & $\mathrm{Cu}$ & $\mathrm{Si}$ & $\mathrm{O}$ & $\mathrm{Cu}$ & $\mathrm{Si}$ & $\mathrm{O}$ \\
\hline Cu-silica-3.0 & $1.4 \pm 0.4$ & $4.3 \pm 0.9$ & $67.5 \pm 1.5$ & $28.1 \pm 0.7$ & $0.3 \pm 0.3$ & $40.1 \pm 0.1$ & $59.6 \pm 31$ \\
Cu-silica-5.4 & $1.5 \pm 0.1$ & $2.6 \pm 0.4$ & $86.5 \pm 2.1$ & $11.0 \pm 1.7$ & $2.6 \pm 2.0$ & $45.9 \pm 0.9$ & $51.5 \pm 6.8$ \\
Cu-silica-10.4 & $31.5 \pm 0.4$ & $36.5 \pm 2.1$ & $37.3 \pm 4.6$ & $26.2 \pm 2.6$ & $19.2 \pm 6.1$ & $22.6 \pm 2.7$ & $58.1 \pm 5.5$ \\
\hline
\end{tabular}

Table 2: weight $\% \mathrm{Cu}$ from ICP-OES, weight $\% \mathrm{Cu}$, Si, and $\mathrm{O}$ from SEM-EDX, and XPS analysis. ${ }^{a}$ analysis representative of entire sample material. ${ }^{b}$ analysis representative of top $10 \mathrm{~nm}$ surface layer. ${ }^{c}$ Values converted from atomic \%, which is provided in Table S1 in the Supplemental Information.

High resolution $\mathrm{Cu} 2 \mathrm{p}_{3 / 2}$ and Si2p atomic orbital XP spectra are displayed in Fig. 4a and $\mathrm{b}$ with the $\mathrm{Cu}$-silica-10.4 spectra on the bottom panels and the Cu-silica-5.4 and -3.0 samples on the top panels (a Si2p spectrum of silica generated from ablating a silicon wafer in water is displayed in the Supplemental Information). $\mathrm{No} \mathrm{Cu}$ was detected in the $\mathrm{Cu} 2 \mathrm{p}_{3 / 2}$ spectrum for the $\mathrm{Cu}$-silica-3.0 sample. In the $\mathrm{Cu} 2 \mathrm{p}_{3 / 2}$ spectra, a peak at $932.7 \mathrm{eV}$ (purple) was deconvoluted in both samples, which corresponds to either $\mathrm{Cu}^{0}(932.6 \mathrm{eV})$ or $\mathrm{Cu}^{+}$as in $\mathrm{Cu}_{2} \mathrm{O}(932.2 \mathrm{eV})$ [43-47]. The second peak around $934 \mathrm{eV}$ in the $\mathrm{Cu}$-silica5.4 sample (blue) corresponds to $\mathrm{Cu}^{2+}$ and matches that of a $\mathrm{CuO}$ species $[42,48,49]$. In the Cu-silica-10.4 sample, this feature it is shifted to $935.3 \mathrm{eV}$ (black), corresponding to $\mathrm{Cu}^{2+}$ interacting with silica [11, 42]. In particular, this feature matches the binding energy of copper phyllosilicate $\left(\mathrm{Cu}_{2} \mathrm{Si}_{2} \mathrm{O}_{5}(\mathrm{OH})_{2}\right.$, Cu-PS) near 935-936 eV [11, 49, 50]. The peaks around 942-945 eV correspond to shake up satellite features from the $2 \mathrm{p} \rightarrow 3 \mathrm{~d}$ transition from the $3 \mathrm{~d}^{9}$ ground state electron configuration of $\mathrm{Cu}^{2+}[43,44,51]$. This feature is strongly present in the $\mathrm{Cu}$-silica-10.4 sample, and only weakly visible in the $\mathrm{Cu}$-silica-5.4 sample due to its $\mathrm{Cu}$ low loading.

The Si2p atomic orbitals in Fig. 4b have several silicon species, with a large peak centered around $103 \mathrm{eV}$ corresponding to oxidized silica, and a small peak near $99 \mathrm{eV}$ (green) corresponding to $\operatorname{Si}^{0}[52,53]$. Within the large oxidized silicon peak around $103 \mathrm{eV}$, two species are deconvoluted for all three samples- 
the peak at $103.5 \mathrm{eV}$ (gray) corresponds to $\mathrm{Si}^{4+}$ of $\mathrm{Si}-\mathrm{O}-\mathrm{Si}$ tetrahedrally coordinated silicon in amorphous silica [53-55], and the feature at $102.2 \mathrm{eV}$ (dark blue) corresponds to $\mathrm{Si}^{3+}$ in the form of $\mathrm{Si}_{2} \mathrm{O}_{3}[54,55]$. A third species was deconvoluted in the $\mathrm{Cu}$-silica-10.4 sample at $100.7 \mathrm{eV}$ (dark green), close to the $101 \mathrm{eV}$ binding energy of $\mathrm{Si}^{+}$[54-56]. Such down-shifting of the $\mathrm{Si}$ binding energy has been attributed to interaction of silica with $\mathrm{Cu}$ atoms $[9,57]$. The up-shifted $\mathrm{Cu}^{2+}$ peak and the down-shifted $\mathrm{Si}^{2+}$ peak suggest an interaction where $\mathrm{Cu}$ is slightly positive and the $\mathrm{Si}$ is slightly negative.

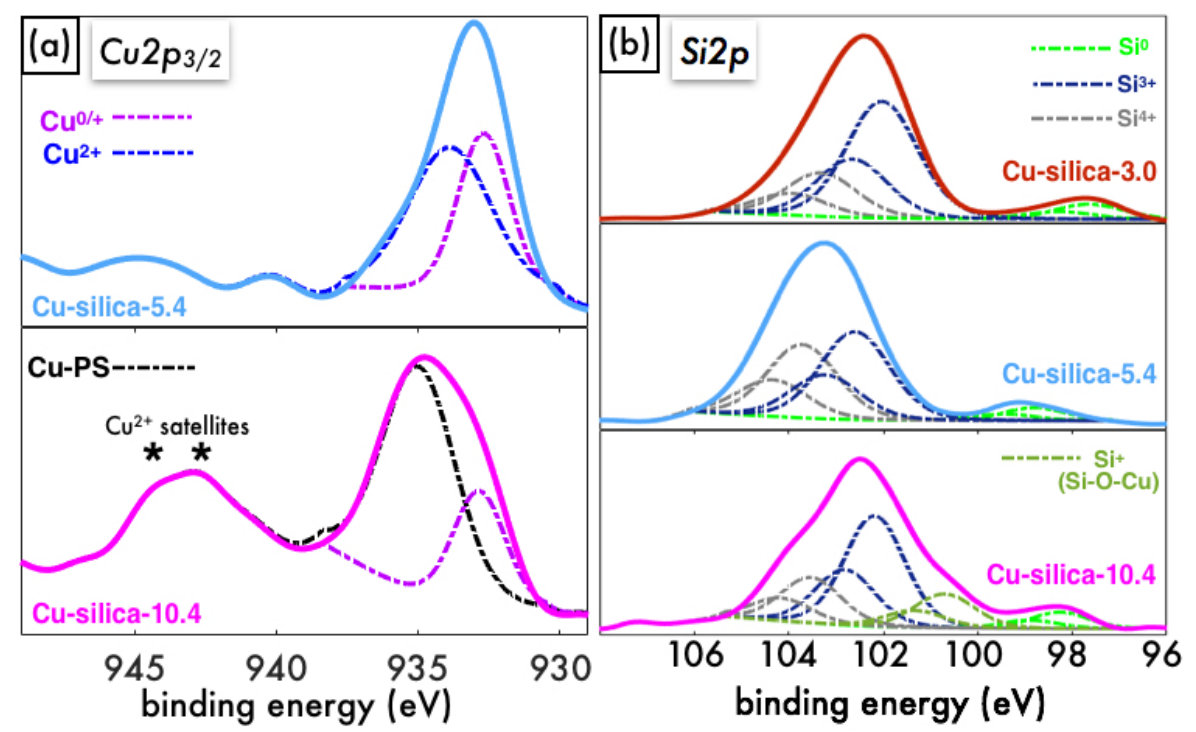

Figure 4: High resolution XP spectra of $\mathrm{Cu} 2 \mathrm{p}_{3 / 2}$ (a) and $\mathrm{Si} 2 \mathrm{p}$ (b) atomic orbitals. Cu-silica samples generated under same experimental conditions, with different $\mathrm{pH}$ solutions.

Figure 5 displays the FTIR spectra of the three samples along with a control silica-10.4 sample, generated by ablating a silicon wafer immersed in water adjusted to $\mathrm{pH} 10.4$ with $\mathrm{KOH}$. The peaks at 800 and $1090 \mathrm{~cm}^{-1}$ in the control sample (gray) correspond to the symmetric and asymmetric stretching of amorphous silica. These two bands are prominent in the $\mathrm{Cu}$-silica-3.0 and -5.4 samples (red and blue), but the $800 \mathrm{~cm}^{-1}$ band is nearly absent $\mathrm{Cu}$-silica-10.4 spectrum (magenta). Moreover, the $1090 \mathrm{~cm}^{-1}$ band is significantly downshifted to $968 \mathrm{~cm}^{-1}$. This shift corresponds to a shift in bonding environment 
from $\mathrm{Si}-\mathrm{O}-\mathrm{Si}$ to $\mathrm{Si}-\mathrm{O}-\mathrm{Cu}$, due to the longer $\mathrm{Cu}-\mathrm{O}$ bond length than $\mathrm{Si}-\mathrm{O}$ [57]. This band is also close to the $1024 \mathrm{~cm}^{-1}$ feature in Cu-PS [6, 58-60]. The $\mathrm{Cu}$-silica-10.4 sample also has a weak feature that could be attributed to $\mathrm{Cu}(\mathrm{OH})_{2}$ at $690 \mathrm{~cm}^{-1}, \mathrm{Cu}-\mathrm{PS}$ at $670 \mathrm{~cm}^{-1}$, or both [6, 58-61]. The broadness of this band and the weak intensity make it difficult to distinguish between these copper structures. The peak around $1400 \mathrm{~cm}^{-1}$ that is present in the $\mathrm{Cu}$ silica-3.0 sample likely arises from the symmetric stretching vibration of nitrate groups in $\mathrm{Cu}_{2} \mathrm{NO}_{3}(\mathrm{OH})$ [62]. All samples have peaks around $1652 \mathrm{~cm}^{-1}$, which corresponds to the $\mathrm{O}-\mathrm{H}$ bending mode of adsorbed water [11, 62].

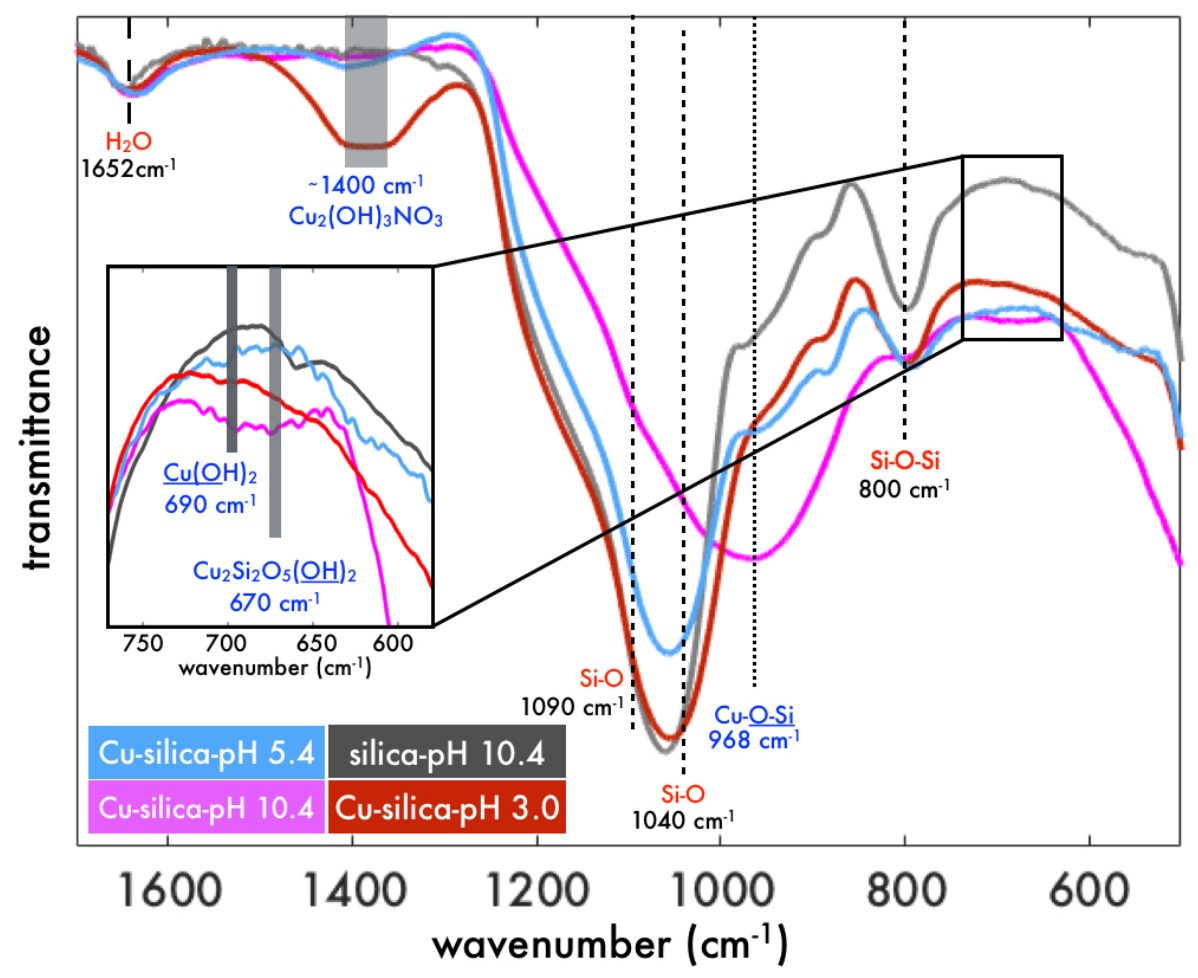

Figure 5: FTIR spectra of silica-pH 10.4 (generated from ablating Si wafer in water at $\mathrm{pH}$ 10.4), the Cu-silica-3.0, Cu-silica-5.4, and $\mathrm{Cu}$-silica-10.4 samples.

XRD patterns of the $\mathrm{Cu}$-silica-10.4 (magenta) and $\mathrm{Cu}$-silica-5.4 (blue) samples are displayed in Fig. 6 compared to a control sample in which a silicon wafer was ablated in water (black). All samples contain sharp, intense peaks located at 
$28.4,47.3,56.1,69.2,76.4$, and $88.12 \theta$ corresponding to the (111), (222), (331), (440), (533), and (640) planes of cubic silicon (ICDD: 04-012-7888). There is a small, broad peak around $362 \theta$ present only in the $\mathrm{Cu}$-silica-10.4 sample that corresponds to either the (111) $\mathrm{CuO}$ plane (35.6 20), the (111) plane of $\mathrm{Cu}_{2} \mathrm{O}$ (36.5 2 $\theta$ ), or a $\mathrm{Cu}-\mathrm{PS}$ structure [6, 58-60]. The inset shows the Gaussian peak fit yielding a FWHM of $4.902 \theta$ corresponding to a $1.78 \mathrm{~nm}$ diameter of the crystalline nanoparticle, according to the Scherrer equation [63]. The FWHM of the fcc-Si (111) peak at $28.42 \theta$ was determined to be $0.252 \theta$, yielding a silicon crystalline diameter of $33.6 \mathrm{~nm}$. The XRD pattern shows that there are crystalline silicon particles present in these samples, consistent with the large spherical particles visible in the TEM images in Fig. 1a. The absence of silica in the XRD patterns supports the amorphous nature of the silica as evident in the HRTEM images, Si2p XPS spectra, and FTIR spectra.

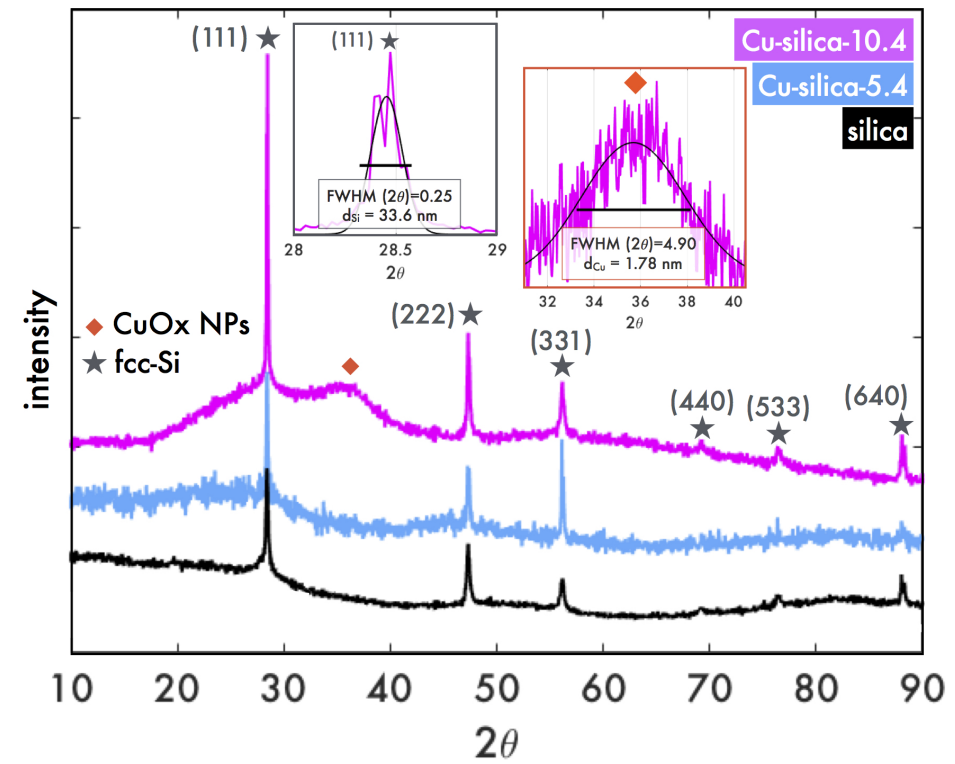

Figure 6: XRD patterns of Cu-silica-10.4, Cu-silica-5.4, and silica generated from laser ablation of silicon wafer in water. 


\subsection{Catalytic Activity}

The catalytic reduction of para-nitrophenol (PNP) by sodium borohydride was employed as a model reaction to compare the catalytic activity of the silica$\mathrm{Cu}$ samples. Because all samples were irradiated under the same laser conditions and underwent the same post-synthesis processing procedure, we assume the amount of silica present in all samples is equivalent, and the rate constants reported reflect the catalytically active copper particles in the samples. Therefore, the same volume of re-dispersed pellet was added to all PNP reactions. Experimental details and calculations for determining the catalytic rate constants have been described in detail elsewhere $[36,38]$ and are provided in the Supplemental Information.

Briefly, the catalytic reduction of PNP to para-aminophenol (PAP) by sodium borohydride follows pseudo-first order reaction kinetics due to the excess of $\mathrm{NaBH}_{4}$ added to the reaction

$$
\mathrm{PNP}+\mathrm{NaBH}_{4(x s)} \stackrel{C u-\text { silica }}{\longrightarrow} \mathrm{PAP}
$$

The para-phenolate ion absorbs strongly at $400 \mathrm{~nm}$, allowing for the reaction rate to be determined by monitoring its absorbance upon the addition of the catalyst. The apparent rate constants, $k_{\text {app }}\left(\mathrm{s}^{-1}\right)$ versus wt.\% $\mathrm{Cu}$ from XPS analysis are displayed in Fig. 7, with the different $\mathrm{pH}$ conditions labeled. There is a linear relationship between the rate constant and the surface $\mathrm{Cu}$ content, reflecting the high catalytic activity of the $\mathrm{Cu}$-silica-10.4 sample which contains the highest amount of surface $\mathrm{Cu}$. While nearly no $\mathrm{Cu}$ was detected in the $\mathrm{Cu}$-silica-3.0 sample, it still possess catalytic activity, suggesting that the there was $\mathrm{Cu}$ present in the sample, but in very small quantities. 


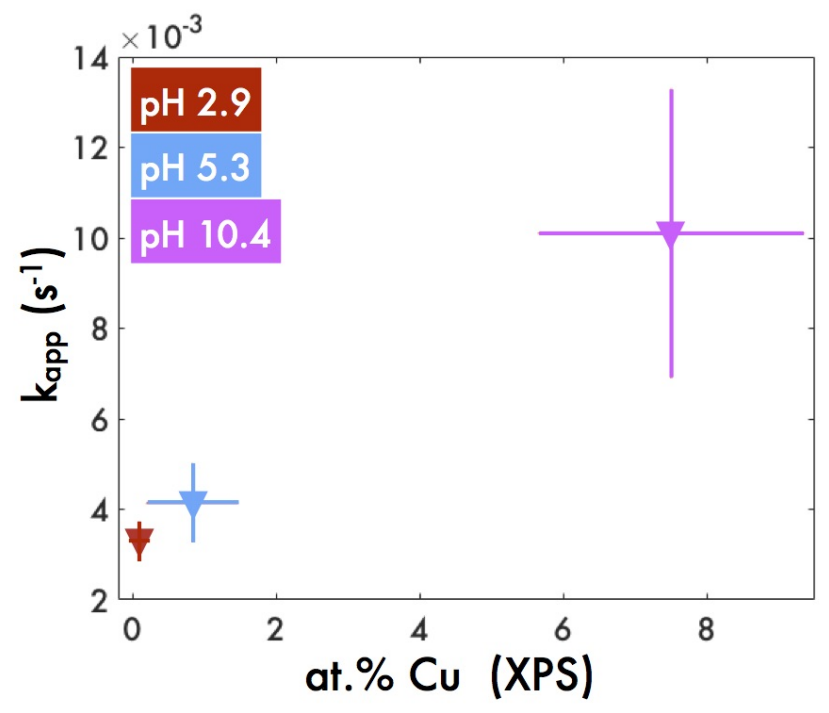

Figure 7: Apparent rate constant ( $\mathrm{k}_{\mathrm{app}}$ ) versus at.\% $\mathrm{Cu}$ from XPS analysis.

\section{Discussion}

Wet chemical approaches to fabricating oxide supported metal nanoparticles emphasize the importance of choosing the support, metal precursor, and solution $\mathrm{pH}$ for maximizing the metal loading [64]. Determining the point of zero charge (PZC) of the support material aids in identifying the $\mathrm{pH}$ conditions for optimal interaction between the metal complex and the oxide support $[64,65]$. The $\mathrm{PZC}$ of a material is the $\mathrm{pH}$ at which the hydroxyl groups that populate the surface of an oxide have a neutral charge. When the $\mathrm{pH}$ is below the PZC of the support, the hydroxyl groups are protonated, and when the $\mathrm{pH}$ is above the PZC, the hydroxyl groups are deprotonated $[13,15,64]$. Silica has a PZC of $\mathrm{pH} 4$, so when the solution $\mathrm{pH}$ is above this value, cationic metal complexes in solution can adsorb onto the negatively charged surface of the silica [15].

The copper loadings reported in Table 2 demonstrate the need for basic $\mathrm{pH}$ to achieve high copper loading under our synthesis conditions; even in the weakly acidic solution at $\mathrm{pH} 5.4$, little copper was found in the product. In contrast, the Cu-silica-10.4 sample had a high copper loading of $31.5 \mathrm{wt} \%$; much 
higher than many previous reports using wet chemical techniques that typically achieve around 10 wt.\% [15, 65-69]. Previous investigations of pH-dependent metal loading on silica that achieved copper loadings above $30 \mathrm{wt} . \%$ have formed $\mathrm{Cu}-\mathrm{PS}$ structures $[10,58,70,71]$, consistent with our results. However, the wet-chemical synthesis methods used are considerably more time- and materialconsuming than our RLAL method. For instance, the method of Toupance et $a l .$, in which a copper nitrate solution adjusted to $\mathrm{pH} 9$ using ammonia mixed with nonporous silica was stirred at room temperature for one week, required 100 times the amount of copper relative to our conditions to yield 36.6 wt. $\% \mathrm{Cu}$ with $4.5 \mathrm{~nm} \mathrm{Cu}$ NPs [58].

In contrast to pre-synthesized fumed silica or nonporous silica spheres used in wet chemical methods, our silica is produced in situ from laser ablation. As the silicon atoms and clusters are ejected into solution, they may interact with other nearby species including hydrated electrons and hydroxyl radicals, resulting in oxidation to silica. Under basic conditions, nearby $\mathrm{OH}^{-}$ions may interact with the oxidized silica clusters, deprotonating their surfaces [72]. The negatively charged silica clusters attract nearby copper in the form of the bridged copper hydroxide dimer, $\left[\mathrm{Cu}_{2}(\mathrm{OH})_{2}\right]^{2+}$, which is formed in the $\mathrm{pH}$ range $6.5-10.5$ $[15,73]$. The abundance of deprotonated silica clusters generated from laser ablation provide numerous sites for these copper complexes to interact with, driving the high copper loading under basic conditions.

Figure 8 displays a graphical representation of the formation mechanisms of the copper-silica materials under the different $\mathrm{pH}$ conditions. We note that the solution $\mathrm{pH}$ decreased from 10.4 to 8.5 and 5.4 to 4.2 during synthesis of the $\mathrm{Cu}$-silica-10.4 and -5.4 samples, respectively. The two samples generated at final solution $\mathrm{pH}$ less than $\mathrm{pH} 4$ are shown above the 'silica PZC 4.0' line [13, 15] in Fig. 8. Under these conditions, silica clusters ablated off of the Si wafer into solution become protonated and repel the surrounding $\mathrm{Cu}^{2+}$ ions, leading to low amounts of $\mathrm{Cu}$ incorporated into the product. While it is possible that a small amount of $\mathrm{Cu}$ in the product dissolves in the acidic solution following laser synthesis (see details in the Supporting Information), this process is unlikely to 
be the primary cause of low $\mathrm{Cu}$ incorporation because little $\mathrm{Cu}$ is incorporated even at an initial $\mathrm{pH}$ of 5.4. For the particles in which $\mathrm{Cu}$ is incorporated into the particles, segregation of the $\mathrm{Cu}$-silica phases result in large $\mathrm{Cu}$-core/silicashell particles with varying sizes and shell thicknesses. The formation of the silica shell rather than silica core may be due to the higher surface energy of silicon than copper in the liquid form. Synthesis of Cu-core/silica-shell particles by evaporating elemental $\mathrm{Cu}$ and $\mathrm{Si}$ using a high powered electron beam resulted in phase segregation with the silicon shell forming around liquid $\mathrm{Cu}$, due to the higher surface energy of liquid silicon relative to liquid $\mathrm{Cu}[74,75]$. In contrast, the proposed formation mechanism of the $\mathrm{Cu}$-silica-10.4 sample is displayed below the line labeled 'silica PZC 4.0', where Cu-O-Si bonds form due to the strong interaction between the deprotonated silica clusters and the cationic copper hydroxide dimers. The morphology contains sheet- and needlelike structures comprised of amorphous silica, copper phyllosilicate, or both, decorated with sub-2 $\mathrm{nm} \mathrm{Cu}$ clusters. The small size of the $\mathrm{Cu}$ particles likely results from the strong interaction between the $\mathrm{Cu}$ nuclei and the silica clusters, halting further $\mathrm{Cu} \mathrm{NP}$ growth. The presence of sub-2 $\mathrm{nm}$ clusters dispersed throughout the silica is similar to our recent report of fs-RLAL synthesis of sub$3 \mathrm{~nm} \mathrm{Au} \mathrm{NPs} \mathrm{dispersed} \mathrm{throughout} \mathrm{a} \mathrm{silica} \mathrm{matrix} \mathrm{under} \mathrm{basic} \mathrm{conditions} \mathrm{[36].}$ Our observation of distinct copper-silica material structures using fs-RLAL at different solution $\mathrm{pH}$ is consistent with previous RLAL studies showing a dependence of Pt-Co NP properties on solution $\mathrm{pH}[29,30]$ and suggests that solution $\mathrm{pH}$ provides a generally applicable method to control nanomaterial properties with RLAL.

\section{Conclusion}

We have synthesized copper-silica nanocomposites using a fs-RLAL approach, with distinct copper-silica morphologies forming from different precursor solution $\mathrm{pH}$ conditions. The highest copper loading on silica of $31.5 \mathrm{wt} . \%$ achieved with a precursor solution $\mathrm{pH}$ of 10.4 generated $1.52 \pm 0.75 \mathrm{~nm} \mathrm{Cu}$ NPs well dis- 


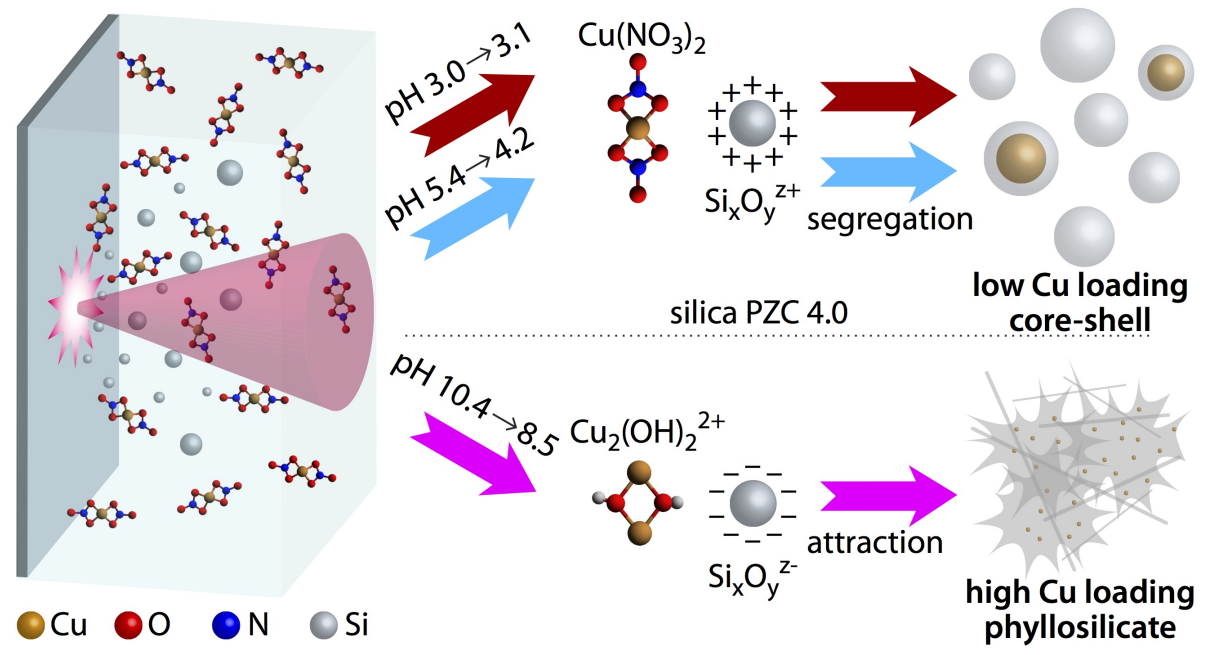

Figure 8: Graphical representation of the $\mathrm{pH}$ dependent formation of $\mathrm{Cu}$-silica samples

persed throughout an amorphous matrix. TEM, XPS, XRD, and FTIR analysis are consistent with the coexistence of copper phyllosilicate and amorphous silica in the matrix. Lower solution $\mathrm{pH}$ of 5.4 and 3.0 resulted in Cu-core/silica-shell morphologies with $\mathrm{Cu}$ NP sizes ranging from $30-80 \mathrm{~nm}$ in diameter, and only 1.4-1.5 wt.\% loading of $\mathrm{Cu}$. The catalytic activity of the synthesized materials was proportional to the copper loading, with very low catalytic activity toward the reduction of para-nitrophenol from the samples synthesized at $\mathrm{pH}$ 3.0 and 5.4, and much higher catalytic activity from the sample synthesized at $\mathrm{pH}$ 10.4. The $\mathrm{pH}$ dependent compositions and morphologies of our copper-silica nanocomposites are attributed to the interaction of positively charged $\mathrm{Cu}$ precursor species and ablated silica species. At low $\mathrm{pH}$, protonation of silica clusters results in copper and silica phase segregation, forming core-shell structures. At high $\mathrm{pH}$, the copper hydroxide dimer ion strongly interacts with deprotonated silica clusters, resulting in sub-2 $\mathrm{nm} \mathrm{Cu}$ clusters supported on a copper phyllosilicate/amorphous silica matrix. The results in this study demonstrate the importance of understanding the effect of solution $\mathrm{pH}$ on the electronic charge of ablated target material to allow for better control over the product morphology. This approach to synthesizing $\mathrm{Cu}$-silica composite nanomaterials provides 
valuable insight into designing RLAL reaction conditions for synthesizing additional metal-oxide nanocomposites with high metal loadings that may be used for catalytic applications.

\section{Acknowledgement}

This work was supported by the American Chemical Society Petroleum Research Fund through Grant 57799-DNI10. Microscopy was performed at the VCU Department of Anatomy and Neurobiology Microscopy Facility, supported by the Higher Education Equipment Trust Fund Grant No. 236160307. We would like to acknowledge the VCU Nanomaterials Core Characterization Facility for additional characterization.

\section{Supplemental Material}

Details of PNP catalytic reactions; Details of HRTEM lattice fringe analysis; Details of XPS composition analysis; additional TEM images; additional XPS spectra of Si2p.

\section{References}

[1] X. Niu, Y. L, J. Tang, Y. Hu, H. Zhao, M. Lan, Electrochemical sensing interfaces with tunable porosity for nonenzymatic glucose detection: A cu foam case, Biosensors and Bioelectronics 51 (2014) 22 - 28. doi:https: //doi.org/10.1016/j.bios.2013.07.032.

[2] A. M. Raspolli Galletti, C. Antonetti, M. Marracci, F. Piccinelli, B. Tellini, Novel microwave-synthesis of cu nanoparticles in the absence of any stabilizing agent and their antibacterial and antistatic applications, Appl. Surf. Sci. 280 (2013) 610 -618. doi:https://doi.org/10.1016/j.apsusc. 2013.05 .035 .

[3] Y. Lee, J. rak Choi, K. J. Lee, N. E. Stott, D. Kim, Large-scale synthesis of copper nanoparticles by chemically controlled reduction for applications of inkjet-printed electronics, Nanotechnology 19 (41) (2008) 415604. doi: 10.1088/0957-4484/19/41/415604.

URL https ://doi.org/10. 1088\%2F0957-4484\%2F19\%2F41\%2F415604 
[4] M. B. Gawande, A. Goswami, F.-X. Felpin, T. Asefa, X. Huang, R. Silva, X. Zou, R. Zboril, R. S. Varma, Cu and Cu-based nanoparticles: Synthesis and applications in catalysis, Chemical Reviews 116 (6) (2016) 3722-3811. doi:10.1021/acs.chemrev.5b00482.

[5] L. Cao, D. Raciti, C. Li, K. J. T. Livi, P. F. Rottmann, K. J. Hemker, T. Mueller, C. Wang, Mechanistic insights for low-overpotential electroreduction of co2 to co on copper nanowires, ACS Catalysis 7 (12) (2017) 8578-8587. doi:10.1021/acscatal.7b03107.

[6] Y. Sheng, H. C. Zeng, Structured assemblages of single-walled 3d transition metal silicate nanotubes as precursors for composition-tailorable catalysts, Chemistry of Materials 27 (3) (2015) 658-667. doi:10.1021/cm502691s.

[7] G. Yin, M. Nishikawa, Y. Nosaka, N. Srinivasan, D. Atarashi, E. Sakai, M. Miyauchi, Photocatalytic carbon dioxide reduction by copper oxide nanocluster-grafted niobate nanosheets, ACS Nano 9 (2) (2015) 2111-2119. doi:10.1021/nn507429e.

[8] L. Zhu, H. Li, Z. Liu, P. Xia, Y. Xie, D. Xiong, Synthesis of the 0d/3d cuo/zno heterojunction with enhanced photocatalytic activity, The Journal of Physical Chemistry C 122 (17) (2018) 9531-9539. doi:10.1021/acs. jpcc.8b01933.

URL https://doi.org/10.1021/acs.jpcc.8b01933

[9] Y. Zhao, J. Zhao, Z. Su, X. Hao, Y. Li, N. Li, Y. Li, Sio2 capsulized cu active nanoparticles: synthesis and activity study, J. Mater. Chem. A 1 (2013) 8029-8036. doi:10.1039/C3TA11281K.

[10] J. Gong, H. Yue, Y. Zhao, S. Zhao, L. Zhao, J. Lv, S. Wang, X. Ma, Synthesis of ethanol via syngas on $\mathrm{cu} / \mathrm{sio} 2$ catalysts with balanced $\mathrm{cu} 0-\mathrm{cu}+$ sites, Journal of the American Chemical Society 134 (34) (2012) 1392213925. doi:10.1021/ja3034153. 
[11] L.-F. Chen, P.-J. Guo, M.-H. Qiao, S.-R. Yan, H.-X. Li, W. Shen, H.-L. $\mathrm{Xu}, \mathrm{K} . \mathrm{N}$. Fan, $\mathrm{Cu} / \mathrm{sio} 2$ catalysts prepared by the ammonia-evaporation method: Texture, structure, and catalytic performance in hydrogenation of dimethyl oxalate to ethylene glycol, Journal of Catalysis 257 (1) (2008) 172 - 180. doi:https://doi.org/10.1016/j.jcat.2008.04.021.

[12] Z. Huang, F. Li, B. Chen, F. Xue, G. Chen, G. Yuan, Nitrogen-rich copolymeric microsheets supporting copper nanoparticles for catalyzing arylation of n-heterocycles, Applied Catalysis A: General 403 (1) (2011) 104 - 111. doi:https://doi.org/10.1016/j.apcata.2011.06.019.

[13] S. Eskandari, G. Tate, N. R. Leaphart, J. R. Regalbuto, Nanoparticle synthesis via electrostatic adsorption using incipient wetness impregnation, ACS Catal. 8 (11) (2018) 10383-10391. doi:10.1021/acscatal.8b03435.

[14] C. Xu, G. Chen, Y. Zhao, P. Liu, X. Duan, L. Gu, G. Fu, Y. Yuan, $\mathrm{N}$. Zheng, Interfacing with silica boosts the catalysis of copper, Nature Commun. 9 (1) (2018) 3367. doi:10.1038/s41467-018-05757-6.

[15] L. Jiao, J. R. Regalbuto, The synthesis of highly dispersed noble and base metals on silica via strong electrostatic adsorption: I. amorphous silica, J. Catal. 260 (2) (2008) 329 - 341. doi :https://doi.org/10.1016/j.jcat. 2008.09 .022 .

[16] D. Zhang, B. Gökce, S. Barcikowski, Laser synthesis and processing of colloids: Fundamentals and applications, Chemical Reviews 117 (5) (2017) 3990-4103. doi:10.1021/acs.chemrev.6b00468.

[17] D. Zhang, J. Liu, P. Li, Z. Tian, C. Liang, Recent advances in surfactantfree, surface-charged, and defect-rich catalysts developed by laser ablation and processing in liquids, ChemNanoMat 3 (8) (2017) 512-533. doi:10.1002/cnma. 201700079.

URL https://onlinelibrary.wiley.com/doi/abs/10.1002/cnma. 201700079 
[18] S. Reichenberger, G. Marzun, M. Muhler, S. Barcikowski, Perspective of surfactant-free colloidal nanoparticles in heterogeneous catalysis, ChemCatChem 11 (18) (2019) 4489-4518. doi:10.1002/cctc. 201900666.

[19] D. Amans, W. Cai, S. Barcikowski, Status and demand of research to bring laser generation of nanoparticles in liquids to maturity, Applied Surface Science 488 (2019) 445 - 454. doi:https://doi.org/10.1016/j .apsusc. 2019.05 .117$.

[20] B. Rethfeld, D. S. Ivanov, M. E. Garcia, S. I. Anisimov, Modelling ultrafast laser ablation, J. Phys. D 50 (19) (2017) 193001. doi:10.1088/1361-6463/ 50/19/193001.

URL https://doi.org/10.1088\%2F $1361-6463 \% 2 \mathrm{~F} 50 \% 2 \mathrm{~F} 19 \% 2 \mathrm{~F} 193001$

[21] M. Dell'Aglio, R. Gaudiuso, O. De Pascale, A. De Giacomo, Mechanisms and processes of pulsed laser ablation in liquids during nanoparticle production, Applied Surface Science 348 (2015) 4 - 9, advanced Synthesis of Functional Nanoparticles by Lasers in Liquids - From Fundamentals to Application in Catalysis, Energy Science, and Biomedicine. doi:https://doi.org/10.1016/j.apsusc. 2015.01.082.

[22] B. Gökce, V. Amendola, S. Barcikowski, Opportunities and challenges for laser synthesis of colloids, ChemPhysChem 18 (9) (2017) 983-985. doi: 10.1002/cphc. 201700310.

[23] J. M. J. Santillán, F. A. Videla, M. B. Fernández van Raap, D. C. Schinca, L. B. Scaffardi, Size dependent cu dielectric function for plasmon spectroscopy: Characterization of colloidal suspension generated by fs laser ablation, Journal of Applied Physics 112 (5) (2012) 054319. doi: $10.1063 / 1.4751328$.

[24] J. M. J. Santillán, F. A. Videla, M. B. Fernández van Raap, D. C. Schinca, L. B. Scaffardi, Analysis of the structure, configuration, and sizing of cu and $\mathrm{cu}$ oxide nanoparticles generated by fs laser ablation of solid target in 
liquids, Journal of Applied Physics 113 (13) (2013) 134305. doi:10.1063/ 1.4798387 .

[25] P. Liu, Z. Li, W. Cai, M. Fang, X. Luo, Fabrication of cuprous oxide nanoparticles by laser ablation in pvp aqueous solution, RSC Adv. 1 (2011) 847-851. doi:10.1039/C1RA00261A.

[26] R. Tilaki, A. Iraji zad, S. Mahdavi, Size, composition and optical properties of copper nanoparticles prepared by laser ablation in liquids, Applied Physics A 88 (2) (2007) 415-419. doi:10.1007/s00339-007-4000-2.

[27] E. Jiménez, K. Abderrafi, J. Martínez-Pastor, R. Abargues, J. L. Valdés, R. Ibáñez, A novel method of nanocrystal fabrication based on laser ablation in liquid environment, Superlattices and Microstructures 43 (5) (2008) 487 - 493, proceedings of the 7th International Conference on Physics of Light-Matter Coupling in Nanostructures. doi:10.1016/j.spmi.2007. 06.025 .

[28] S. Hu, G. Goenaga, C. Melton, T. A. Zawodzinski, D. Mukherjee, Ptco/coox nanocomposites: Bifunctional electrocatalysts for oxygen reduction and evolution reactions synthesized via tandem laser ablation synthesis in solution-galvanic replacement reactions, Applied Catalysis B: Environmental 182 (2016) 286 - 296. doi:10.1016/j.apcatb.2015.09.035.

[29] S. Hu, M. Tian, E. L. Ribeiro, G. Duscher, D. Mukherjee, Tandem laser ablation synthesis in solution-galvanic replacement reaction (lasis-grr) for the production of ptco nanoalloys as oxygen reduction electrocatalysts, J. Power Sources 306 (2016) 413-423. doi:10.1016/j.jpowsour.2015.11. 078.

[30] S. Hu, K. Cheng, E. L. Ribeiro, K. Park, B. Khomami, D. Mukherjee, A facile and surfactant-free route for nanomanufacturing of tailored ternary nanoalloys as superior oxygen reduction reaction electrocatalysts, Catal. Sci. Technol. 7 (2017) 2074-2086. doi:10.1039/C7CY00073A. 
[31] B. M. Hunter, J. D. Blakemore, M. Deimund, H. B. Gray, J. R. Winkler, A. M. Müller, Highly active mixed-metal nanosheet water oxidation catalysts made by pulsed-laser ablation in liquids, J. Am. Chem. Soc. 136 (38) (2014) 13118-13121. doi:10.1021/ja506087h.

URL https://doi.org/10.1021/ja506087h

[32] B. M. Hunter, W. Hieringer, J. R. Winkler, H. B. Gray, A. M. Müller, Effect of interlayer anions on [nife]-ldh nanosheet water oxidation activity, Energy Environ. Sci. 9 (5) (2016) 1734-1743. doi:10.1039/C6EE00377J. URL http://dx.doi.org/10.1039/C6EE00377 J

[33] C. W. Roske, J. W. Lefler, A. M. Müller, Complex nanomineral formation utilizing kinetic control by plal, Journal of Colloid and Interface Science 489 (2017) 68 - 75, laser Synthesis. doi:https://doi.org/10.1016/j. jcis.2016.08.079.

[34] E. Jiménez, K. Abderrafi, R. Abargues, J. L. Valdés, J. P. Martínez-Pastor, Laser-ablation-induced synthesis of sio2-capped noble metal nanoparticles in a single step, Langmuir 26 (10) (2010) 7458-7463. doi:10.1021/ la904179x.

[35] V. A. Ermakov, E. Jimenez-Villar, J. M. C. d. Silva Filho, E. Yassitepe, N. V. V. Mogili, F. Iikawa, G. F. de Sá, C. L. Cesar, F. C. Marques, Size control of silver-core/silica-shell nanoparticles fabricated by laser-ablationassisted chemical reduction, Langmuir 33 (9) (2017) 2257-2262. doi:10. 1021/acs. langmuir.6b04308.

[36] M. G. John, K. M. Tibbetts, One-step femtosecond laser ablation synthesis of sub-3âẮnm gold nanoparticles stabilized by silica, Applied Surface Science 475 (2019) 1048 - 1057. doi:https://doi.org/10.1016/j.apsusc. 2019.01 .042 .

[37] P. Hervés, M. Pérez-Lorenzo, L. M. Liz-Marzán, J. Dzubiella, Y. Lu, M. Ballauff, Catalysis by metallic nanoparticles in aqueous solution: model reactions, Chem. Soc. Rev. 41 (2012) 5577. doi:10.1039/C2CS35029G. 
[38] S. Wunder, F. Polzer, Y. Lu, Y. Mei, M. Ballauff, Kinetic analysis of catalytic reduction of 4-nitrophenol by metallic nanoparticles immobilized in spherical polyelectrolyte brushes, J. Phys. Chem. C 114 (19) (2010) 88148820. doi:10.1021/jp101125j.

[39] V. K. Meader, M. G. John, C. J. Rodrigues, K. M. Tibbetts, Roles of free electrons and h2o2 in the optical breakdown-induced photochemical reduction of aqueous [aucl4]âĹS, The Journal of Physical Chemistry A 121 (36) (2017) 6742-6754. doi:10.1021/acs .jpca.7b05370.

[40] V. K. Meader, M. G. John, L. M. Frias Batista, S. Ahsan, K. M. Tibbetts, Radical chemistry in a femtosecond laser plasma: Photochemical reduction of ag + in liquid ammonia solution, Molecules 23 (3). doi: $10.3390 / \mathrm{molecules} 23030532$.

URL https://www.mdpi.com/1420-3049/23/3/532

[41] T. A. Lastovina, A. P. Budnyk, G. A. Khaishbashev, E. A. Kudryavtsev, A. V. Soldatov, Copper-based nanoparticles prepared from copper (ii) acetate bipyridine complex, J. Serbian Chem. Soc. 81 (7) (2016) 751-762.

[42] X. Wang, K. Ma, L. Guo, Y. Tian, Q. Cheng, X. Bai, J. Huang, T. Ding, $\mathrm{X} . \mathrm{Li}, \mathrm{Cu} / \mathrm{zno} /$ sio2 catalyst synthesized by reduction of zno-modified copper phyllosilicate for dimethyl ether steam reforming, Applied Catalysis A: General 540 (2017) 37 - 46. doi:https://doi.org/10.1016/j.apcata. 2017.04 .013$.

[43] M. C. Biesinger, Advanced analysis of copper x-ray photoelectron spectra, Surf. Interf. Anal. 49 (13) (2017) 1325-1334. doi:10.1002/sia.6239.

[44] M. C. Biesinger, L. W. Lau, A. R. Gerson, R. S. Smart, Resolving surface chemical states in xps analysis of first row transition metals, oxides and hydroxides: Sc, ti, v, cu and zn, Applied Surface Science 257 (3) (2010) 887 - 898. doi:https://doi.org/10.1016/j.apsusc.2010.07.086. 
[45] M. Marques, A. Ferraria, J. Correia, A. Botelho do Rego, R. Vilar, Xrd, xps and sem characterisation of cu-nbc nanocomposite produced by mechanical alloying, Materials Chemistry and Physics 109 (1) (2008) 174 - 180. doi: https://doi.org/10.1016/j.matchemphys.2007.10.032.

[46] Y. H. Kim, D. K. Lee, H. G. Cha, C. W. Kim, Y. C. Kang, Y. S. Kang, Preparation and characterization of the antibacterial cu nanoparticle formed on the surface of sio2 nanoparticles, The Journal of Physical Chemistry B 110 (49) (2006) 24923-24928. doi:10.1021/jp0656779.

[47] J. F. Moulder, W. F. Stickle, P. E. Sobol, K. D. Bomben, Handbook of Xray Photoelectron Spectroscopy, Perkin-Elmer Corp., Physical Electronics Division, 1979.

[48] D. Tahir, S. Tougaard, Electronic and optical properties of $\mathrm{cu}, \mathrm{CuO}$ and cu2o studied by electron spectroscopy, Journal of Physics: Condensed Matter 24 (17) (2012) 175002. doi:10.1088/0953-8984/24/17/175002.

[49] R.-P. Ye, L. Lin, J.-X. Yang, M.-L. Sun, F. Li, B. Li, Y.-G. Yao, A new low-cost and effective method for enhancing the catalytic performance of cu-sio 2 catalysts for the synthesis of ethylene glycol via the vapor-phase hydrogenation of dimethyl oxalate by coating the catalysts with dextrin, Journal of Catalysis 350 (2017) 122-132. doi:10.1016/j.jcat.2017.02. 018.

[50] X. Li, J. Zhang, M. Zhang, W. Zhang, M. Zhang, H. Xie, Y. Wu, Y. Tan, The support effects on the direct conversion of syngas to higher alcohol synthesis over copper-based catalysts, Catalysts 9 (2). doi:10.3390/ catal9020199.

[51] X. Huang, M. Ma, S. Miao, Y. Zheng, M. Chen, W. Shen, Hydrogenation of methyl acetate to ethanol over a highly stable cu/sio2 catalyst: Reaction mechanism and structural evolution, Applied Catalysis A: General 531 (2017) 79 -88. doi:https://doi.org/10.1016/j.apcata.2016.12.006. 
[52] S. Dahle, L. Wegewitz, F. Qi, A. Weber, W. Maus-Friedrichs, Silicon dioxide coating of titanium dioxide nanoparticles from dielectric barrier discharge in a gaseous mixture of silane and nitrogen, Plasma Chemistry and Plasma Processing 33. doi:10.1007/s11090-013-9472-6.

[53] F. Lamastra, S. Mori, V. Cherubini, M. Scarselli, F. Nanni, A new green methodology for surface modification of diatomite filler in elastomers, Materials Chemistry and Physics 194 (2017) 253-260. doi:10.1016/j. matchemphys. 2017.03.050.

[54] J. Won Ma, W.-J. Lee, J. M. Bae, K. Jeong, S. Hoon Oh, J.-H. Kim, S.-h. Kim, J.-H. Seo, J.-P. Ahn, H. Kim, M.-H. Cho, Carrier mobility enhancement of tensile strained si and sige nanowires via surface defect engineering, Nano letters 15. doi:10.1021/acs.nanolett.5b01634.

[55] A. Al-Kattan, Y. Ryabchikov, T. Baati, V. Chirvony, J. F. Sánchez Royo, M. Sentis, D. Braguer, V. Timoshenko, M.-A. Estève, A. V. Kabashin, Ultrapure laser-synthesized si nanoparticles with variable oxidation state for biomedical applications, J. Mater. Chem. B 4 (2016) 7852-7858. doi: 10. 1039/C6TB02623K.

[56] M. Bashouti, K. Sardashti, J. Ristein, S. Christiansen, Early stages of oxide growth in h-terminated silicon nanowires: Determination of kinetic behavior and activation energy, Physical chemistry chemical physics : PCCP 14 (2012) 11877-81. doi:10.1039/c2cp41709j.

[57] Y. Kong, H. Zhu, G. Yang, X. Guo, W. Hou, Q. Yan, M. Gu, C. Hu, Investigation of the structure of mcm- 41 samples with a high copper content, Advanced Functional Materials 14 (8) (2004) 816-820. doi: 10.1002/adfm. 200305111.

[58] T. Toupance, M. Kermarec, J.-F. Lambert, C. Louis, Conditions of formation of copper phyllosilicates in silica-supported copper catalysts prepared by selective adsorption, The Journal of Physical Chemistry B 106 (9) (2002) 2277-2286. doi:10.1021/jp013153x. 
[59] W. Di, J. Cheng, S. Tian, J. Li, J. Chen, Q. Sun, Synthesis and characterization of supported copper phyllosilicate catalysts for acetic ester hydrogenation to ethanol, Applied Catalysis A: General 510 (2016) $244-259$. doi:https://doi.org/10.1016/j.apcata.2015.10.026.

[60] Z.-Q. Wang, Z.-N. Xu, S.-Y. Peng, M.-J. Zhang, G. Lu, Q.-S. Chen, Y. Chen, G.-C. Guo, High-performance and long-lived cu/sio2 nanocatalyst for co2 hydrogenation, ACS Catalysis 5 (7) (2015) 4255-4259. doi: 10.1021/acscatal.5b00682.

[61] Z. Huang, F. Cui, H. Kang, J. Chen, X. Zhang, C. Xia, Highly dispersed silica-supported copper nanoparticles prepared by precipitationấ̌́Sgel method: A simple but efficient and stable catalyst for glycerol hydrogenolysis, Chemistry of Materials 20 (15) (2008) 5090-5099. doi:10.1021/cm8006233.

[62] H. Li, L. Ban, Z. Wang, P. Meng, Y. Zhang, R. Wu, Y. Zhao, Regulation of cu species in cuo/sio2 and its structural evolution in ethynylation reaction, Nanomaterials 9 (842). doi:10.3390/nano9060842.

[63] A. Monshi, M. R. Foroughi, M. Monshi, Modified scherrer equation to estimate more accurately nano-crystallite size using xrd, World Journal of Nano Science and Engineering 2 (2012) 154-160. doi:10.4236/wjnse. 2012.23020.

[64] P. Mäki-Arvela, D. Y. Murzin, Effect of catalyst synthesis parameters on the metal particle size, Appl. Cat. A: General 451 (2013) 251 - 281. doi: https://doi.org/10.1016/j.apcata.2012.10.012.

[65] A. Wong, Q. Liu, S. Griffin, A. Nicholls, J. R. Regalbuto, Synthesis of ultrasmall, homogeneously alloyed, bimetallic nanoparticles on silica supports, Science 358 (6369) (2017) 1427-1430. doi:10.1126/science.aao6538.

[66] L. Trouillet, T. Toupance, F. Villain, C. Louis, In situ characterization of the coordination sphere of cuii complexes supported on silica during the 
preparation of $\mathrm{cu} / \mathrm{sio} 2$ catalysts by cation exchange, Phys. Chem. Chem. Phys. 2 (2000) 2005-2014. doi:10.1039/A909427J.

[67] E. Guerreiro, O. Gorriz, G. Larsen, L. Arrúa, Cu/sio2 catalysts for methanol to methyl formate dehydrogenation: A comparative study using different preparation techniques, Applied Catalysis A: General 204 (1) (2000) 33 - 48. doi:https://doi.org/10.1016/S0926-860X (00) 00507-X.

[68] C. Rudolf, F. Abi-Ghaida, B. Dragoi, A. Ungureanu, A. Mehdi, E. Dumitriu, An efficient route to prepare highly dispersed metallic copper nanoparticles on ordered mesoporous silica with outstanding activity for hydrogenation reactions, Catal. Sci. Technol. 5 (2015) 3735-3745. doi: 10.1039/C5CY00428D.

[69] N. H. Khdary, M. A. Ghanem, M. E. Abdesalam, M. M. Al-Garadah, Sequestration of co2 using cu nanoparticles supported on spherical and rodshape mesoporous silica, Journal of Saudi Chemical Society 22 (3) (2018) 343 - 351. doi:https://doi.org/10.1016/j.jscs. 2016.05.004.

[70] H. Yue, Y. Zhao, S. Zhao, B. Wang, X. Ma, J. Gong, A copper-phyllosilicate core-sheath nanoreactor for carbon-oxygen hydrogenolysis reactions, Nature Communications 4 (2013) $2339 \mathrm{EP}-$.

[71] C. van der Grift, P. Elberse, A. Mulder, J. Geus, Preparation of silicasupported copper catalysts preparation of silica-supported copper catalysts preparation of silica-supported copper catalysts by means of depositionprecipitation, Appl. Cat. 59 (1990) 275-289.

[72] F. K. Crundwell, On the mechanism of the dissolution of quartz and silica in aqueous solutions, ACS Omega 2 (3) (2017) 1116-1127. doi:10.1021/ acsomega. $7 \mathrm{~b} 00019$.

[73] M. Schreier, S. Teren, L. Belcher, J. Regalbuto, J. Miller, The nature of 
'overexchanged' copper and platinum on zeolites, Nanotechnology 16 (2005)

S582-91. doi:10.1088/0957-4484/16/7/036.

[74] A. V. Nomoev, S. P. Bardakhanov, M. Schreiber, D. G. Bazarova, N. A. Romanov, B. B. Baldanov, B. R. Radnaev, V. V. Syzrantsev, Structure and mechanism of the formation of core-shell nanoparticles obtained through a one-step gas-phase synthesis by electron beam evaporation, Beilstein J. Nanotechnol. 6 (2015) 874-880.

[75] N. Eustathopoulos, B. Drevet, Surface tension of liquid silicon: High or low value?, Journal of Crystal Growth 371 (2013) 77 - 83. doi:https: //doi.org/10.1016/j.jcrysgro.2013.02.010. 